\title{
Le système de santé en mutation - zoom sur les tarifs
}

Kerstin Schutz

Susanne Christen

Département Tarifs et conventions pour la médecine ambulatoire en Suisse
Le 23 octobre 2013, le domaine Tarifs et conventions pour la médecine ambulatoire en Suisse de la FMH a eu le plaisir de réunir une centaine de délégués tarifaires et invités engagés à Berne.

Placée sous la devise «Le système de santé en mutation - zoom sur les tarifs», cette journée a été l'occasion de revenir entre autres sur l'intervention imminente de la Confédération dans la structure tarifaire TARMED. Le Dr Ignazio Cassis, conseiller national et président de «curafutura», a ouvert la séance avec un exposé porteur d'un message sans équivoque: le partenariat tarifaire n'est pas un modèle dépassé!

Ensuite, le Dr Jürg Schlup, président de la $\mathrm{FMH}$, et le Dr Ernst Gähler ont fait le point sur la situation actuelle dans le domaine des tarifs ambulatoires.

Durant l'après-midi, la FMH, H+ et la CTM ont présenté leurs travaux communs dans le cadre de la révision du TARMED. Alors que les premières équipes de spécialistes sont sur le point de terminer leurs travaux et que d'autres se mettent à l'œuvre, l'objectif est de terminer la révision sur le plan technique d'ici fin 2015.

Hormis les exposés en plénum, les délégués tarifaires et les invités ont eu l'occasion de participer à trois ateliers:

- Lors de l'atelier «L'organe de médiation», Morena Hostettler Socha, lic. iur., avocate et ombudsman de l'assurancemaladie, et le Dr Philippe Luchsinger, ombudsman de la société des médecins du canton de Zurich, ont permis aux participants de se familiariser avec l'activité de médiateur. Les services de médiation contribuent notamment à réduire le nombre de procédures juridiques en dissipant les malentendus entre les caisses-maladie et les assurés ou entre les médecins et leurs patients, en cherchant des explications et en leur fournissant des informations.

- Les Drs Philip Moline et Renato Laffranchi de Newindex ont quant à eux consacré leur atelier au processus de traitement des données collectées auprès des médecins et répondu aux questions suivantes: comment garantir la protection des données et pourquoi la livraison des données à un centre de confiance est-elle importante pour l'ensemble des médecins? Les données peuvent-elles dire si l'augmentation des factures traitées selon le système du tiers payant a des répercussions sur les valeurs du point tarifaire?

- Enfin, pour la première fois, un atelier destiné aux nouveaux délégués tarifaires a été organisé. Thomas Kessler, collaborateur du domaine Tarifs et conventions pour le domaine ambulatoire de la $\mathrm{FMH}$, a notamment expliqué aux participants où trouver les informations importantes concernant les tarifs ambulatoires, comment la prestation médicale est calculée, quels sont les différents paramètres d'une position tarifaire et ce qui se cache réellement derrière.

Vous trouverez de plus amples informations concernant cette journée ainsi que toutes les présentations PowerPoint des exposés et ateliers sur le site internet de la FMH. (www.fmh.ch $\rightarrow$ Tarifs ambulatoires $\rightarrow \mathrm{Pu}$ blications $\rightarrow$ Journée des délégués tarifaires) 

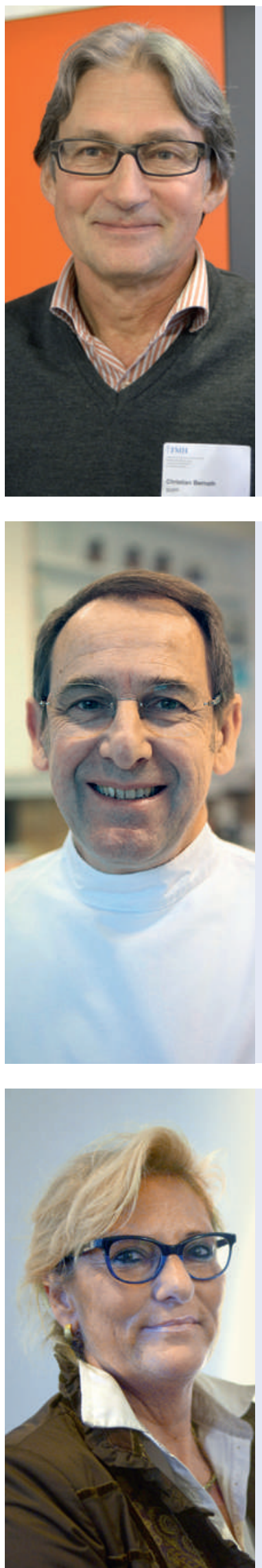

Nous avons également profité de cette journée pour interroger quelques participants sur des questions actuelles liées aux tarifs:

\section{Dr Christian Bernath, membre du Comité et président de la Commission permanente} des tarifs de la Société suisse de psychiatrie et psychothérapie (SSPP)

Votre pronostic pour les cinq prochaines années: tarifs étatiques ou autonomie tarifaire? Le partenariat tarifaire doit être maintenu coûte que coûte. Ce n'est qu'ainsi que les connaissances spécifiques du corps médical pourront être intégrées au tarif et qu'on disposera d'un tarif approprié. Ce sont précisément ces connaissances qui font défaut aux organes de l'Etat.

Vos souhaits et remarques concernant la Journée des délégués tarifaires?

L'objectif de cette journée vise à informer les délégués tarifaires de l'évolution actuelle des tarifs et de l'avancement des négociations tarifaires, à approfondir leurs connaissances concernant les tarifs et à fournir des réponses à leurs questions. Il est particulièrement intéressant de pouvoir entendre l'avis de personnes extérieures au monde de la santé: politiciens, représentants de l'OFSP et des assureurs, éthiciens, économistes de la santé, etc.

Qu'attendez-vous de la réorganisation de TARMED Suisse?

TARMED Suisse doit redevenir opérationnel et j'attends donc de toutes les parties concernées qu'elles s'intéressent sérieusement au développement du TARMED. Les principaux acteurs de la santé doivent y être représentés. Le principe d'unanimité ouvre grand la porte à l'arbitraire et doit impérativement être supprimé.

\section{Dr Jean-Pierre Grillet, Société suisse de dermatologie et vénéréologie}

Qu'attendez-vous de la révision globale du TARMED?

Le tarif a une importance majeure dans la qualité des soins. Les techniques non rentables sont abandonnées au profit des prestations bien rétribuées. Nous attendons de la révision du TARMED un réajustement des positions dans un esprit constructif. Dans sa conception actuelle, le TARMED est relativement bien équilibré. Par conséquent, la révision ne doit pas conduire à une baisse globale des revenus dans une spécialité, mais doit respecter un équilibre dans la pratique de toutes les spécialités.

Comment percevez-vous la collaboration entre votre société et le domaine Tarifs et conventions ambulatoires de la FMH - quelles attentes avez-vous?

Nous avons eu jusqu'ici de très bonnes relations avec le service tarifaire, mais les discussions de détails n'ont pas encore eu lieu. Nous souhaitons pouvoir démontrer que la prise en charge des patients présentant des affections dermatologiques ne peut se concevoir que dans une prise en charge globale du patient et que cette activité ne peut pas se résumer à un coup d'œil, diagnostic et prescription.

Votre pronostic pour les cinq prochaines années: tarifs étatiques ou autonomie tarifaire?

Dans cinq ans, nous serons probablement sous un régime de caisse-maladie publique, avec des tarifs très inférieurs aux tarifs actuels et une dette de plusieurs milliards. Un partenariat tarifaire ne sera possible que dans un système de consultation ambulatoire avec tarif privé en parallèle au système étatique. Un glissement vers une médecine à deux vitesses semble probablement incontournable.

\section{Dr Karin Julia Stadlin, présidente de la société des médecins du canton de Zoug}

Qu'attendez-vous de la réorganisation de TARMED Suisse?

L'autonomie tarifaire est primordiale! Cela suppose une entente entre les partenaires, faute de quoi ce sera le Conseil fédéral qui décidera et l'on se dirigera alors tout droit vers une «médecine étatique à crédit»!

Le principal changement chez TARMED Suisse doit être la prise de décisions à la majorité et non plus à l'unanimité comme c'est le cas maintenant. Il faut également un droit de véto, donc un système de «majorité avec droit de véto». Concernant la collaboration entre la FMH, santésuisse, la CTM, H+ et la CDS, il faut mettre l'accent sur les structures tarifaires et non sur les prix. L'objectif est que la révision tarifaire de la FMH soit acceptée par tous.

Vos souhaits et remarques concernant la Journée des délégués tarifaires?

J'attends de la Journée des délégués tarifaires qu'elle aborde à la fois la situation politique actuelle et à l'avenir (par ex. les tarifs médicaux en 2020?). 


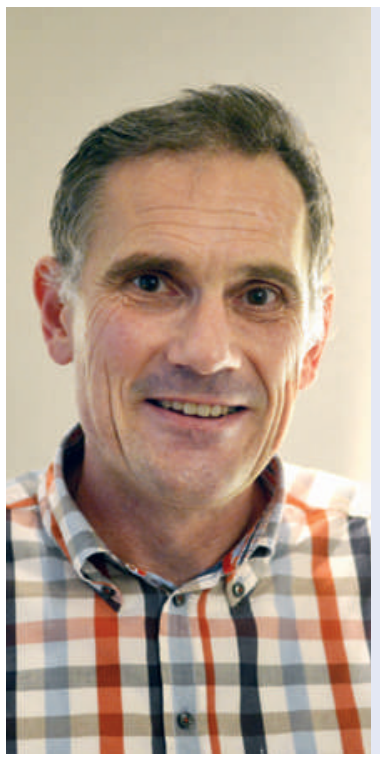

\section{Dr Martin Tönz, délégué tarifaire de la Société suisse de chirurgie pédiatrique}

Qu'attendez-vous de la révision globale du TARMED?

L'objectif du TARMED était et reste de permettre la prise en compte correcte des prestations médicales. La version actuelle du TARMED ne remplit cependant que partiellement cet objectif; dans différents domaines, notamment en chirurgie pédiatrique, ce n'est en effet pas le cas. En outre, le TARMED se fonde sur des données économiques qui ne correspondent plus à la réalité. Ces éléments devront donc être pris en compte dans la nouvelle version.

Les valeurs intrinsèques sont l'un des sujets les plus brûlants du TARMED. La modification de ces paramètres est en effet perçue comme une mesure politique possible en vue de redistribuer les revenus aux médecins de premier recours. Est-ce une mesure appropriée selon vous?

Non, en aucun cas. C'est une mesure qui va dénaturer le TARMED au point de le rendre inutilisable. Autant se faire engager par l'Etat avec un salaire horaire fixe. Une médecine étatique ne permet en aucun cas de résoudre les problèmes des médecins de premier recours.

Comment percevez-vous la collaboration entre votre société et le domaine Tarifs et conventions ambulatoires de la FMH - quelles attentes avez-vous?

Pour moi, la collaboration doit se fonder sur un dialogue constructif afin de permettre la prise en compte correcte des prestations de chirurgie pédiatrique grâce aux connaissances spécifiques de chaque partenaire.

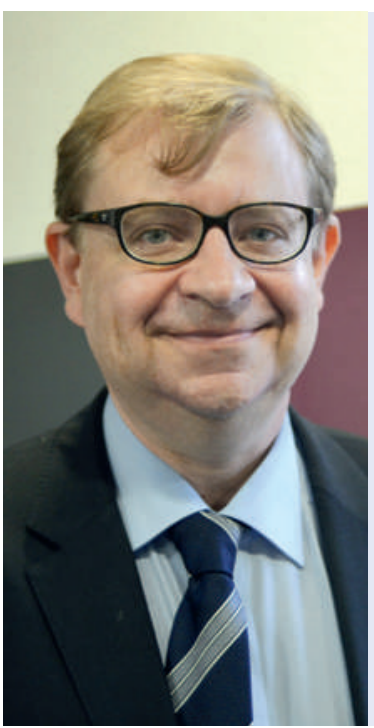

\section{Dr Markus Trutmann, secrétaire général de la fmch}

Les valeurs intrinsèques sont l'un des sujets les plus brûlants du TARMED. La modification de ces paramètres est en effet perçue comme une mesure politique possible en vue de redistribuer les revenus aux médecins de premier recours. Est-ce une mesure appropriée selon vous?

Le TARMED n'a pas été conçu pour redistribuer les revenus. Les valeurs intrinsèques doivent soit être conservées soit totalement supprimées. Les réductions linéaires n'ont pas lieu d'être car elles sont contraires à la LAMal.

Que pensez-vous du nouvel acteur «curafutura» - est-ce une chance ou un inconvénient d'avoir toujours plus d'organisations?

curafutura est une chance. Sa charte est purement libérale. Mais ce qui est plus important encore, c'est la présence d'une nouvelle communauté d'achats en plus de tarifsuisse, à savoir HSK. Le manque de concurrence dans le cadre de l'achat de prestations était en effet l'un des plus grands malentendus dans le secteur de l'assurance-maladie. Grâce à ces nouveaux acteurs, il y a enfin du mouvement.

Votre pronostic pour les cinq prochaines années: tarifs étatiques ou autonomie tarifaire?

Tout indique une présence accrue de l'Etat dans notre système de santé. Et les tarifs ne sont pas une exception. Pour les médecins, les hôpitaux et les assurances qui défendent une pensée entrepreneuriale, cela signifie qu'il faut chercher de nouvelles options en dehors de la LAMal. Et le plus tôt sera le mieux.

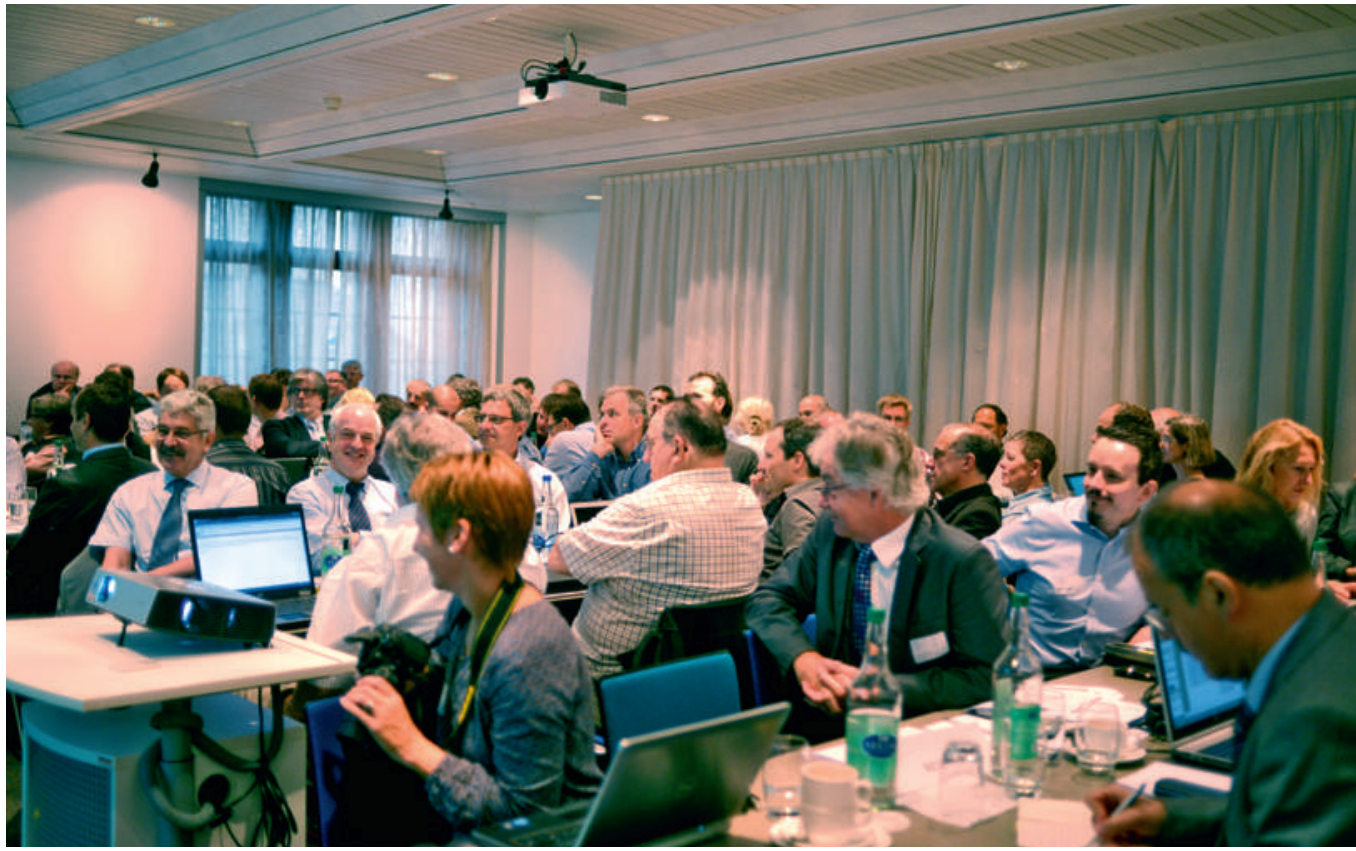

Nous tenons à remercier tous les délégués pour leur participation et les discussions constructives. Prochaine Journée des délégués tarifaires: mercredi 2 avril 2014, Hôtel Ador, Berne

Contact:

FMH

Tarifs et conventions pour la médecine ambulatoire en Suisse Frohburgstrasse 15 CH-4600 Olten tarife.ambulant[at]fmh.ch 